\title{
Human neuroblastoma cells with acquired resistance to the p53 activator RITA retain functional p53 and sensitivity to other p53 activating agents
}

\author{
M Michaelis ${ }^{1,10}$, F Rothweiler ${ }^{1}$, B Agha ${ }^{1}$, S Barth ${ }^{1,2}$, Y Voges ${ }^{1}$, N Löschmann ${ }^{1}$, A von Deimling ${ }^{3,4}$, R Breitling ${ }^{5,6}$, H Wilhelm Doerr ${ }^{1}$, \\ F Rödel $^{7}$, D Speidel ${ }^{8,9}$ and $\mathrm{J}$ Cinatl $\mathrm{Jr}^{*, 1}$
}

Adaptation of wild-type p53 expressing UKF-NB-3 cancer cells to the murine double minute 2 inhibitor nutlin-3 causes de novo p53 mutations at high frequency (13/20) and multi-drug resistance. Here, we show that the same cells respond very differently when adapted to RITA, a drug that, like nutlin-3, also disrupts the p53/Mdm2 interaction. All of the 11 UKF-NB-3 sub-lines adapted to RITA that we established retained functional wild-type p53 although RITA induced a substantial p53 response. Moreover, all RITA-adapted cell lines remained sensitive to nutlin-3, whereas only five out of 10 nutlin-3-adapted cell lines retained their sensitivity to RITA. In addition, repeated adaptation of the RITA-adapted sub-line UKF-NB-3 ${ }^{r}$ ITA ${ }^{10 \mu M}$ to nutlin-3 resulted in p53 mutations. The RITA-adapted UKF-NB-3 sub-lines displayed no or less pronounced resistance to vincristine, cisplatin, and irradiation than nutlin-3-adapted UKF-NB-3 sub-lines. Furthermore, adaptation to RITA was associated with fewer changes at the expression level of antiapoptotic factors than observed with adaptation to nutlin-3. Transcriptomic analyses indicated the RITAadapted sub-lines to be more similar at the gene expression level to the parental UKF-NB-3 cells than nutlin-3-adapted UKF-NB-3 sub-lines, which correlates with the observed chemotherapy and irradiation sensitivity phenotypes. In conclusion, RITA-adapted cells retain functional p53, remain sensitive to nutlin-3, and display a less pronounced resistance phenotype than nutlin-3adapted cells.

Cell Death and Disease (2012) 3, e294; doi:10.1038/cddis.2012.35; published online 5 April 2012

Subject Category: Cancer

p53 is activated in response to DNA damage and other stress stimuli and can cause cell-cycle inhibition, DNA repair, and cell death, respectively. Most cytotoxic anticancer drugs exert their effects under the involvement of p53 activation if the functional gene is expressed, which is the case in about $50 \%$ of all tumours. In addition, a number of compounds that were designed to activate p53 in cancer cells without causing DNA damage ('non-genotoxic p53 activators') are under preclinical and clinical investigation as anticancer drugs. ${ }^{1,2}$ Given that therapeutically applied DNA damage may favour chromosomal instability, mutations, and resistance formation and also support the development of secondary malignancies through introduction of additional mutations, therapeutic strategies that induce apoptosis and growth arrest in a non-genotoxic way have been considered very promising.

Some of the non-genotoxic p53 activators, including the molecules nutlin-3 and RITA, interfere with the interaction of p53 and its endogenous regulator murine double minute 2 (MDM2, in humans also called HDM2). MDM2 is a ubiquitin E3 ligase that binds to p53. Thereby, MDM2 impairs the transactivation function of p53 and targets it for proteasomal degradation. Consequently, small molecules that impair or disrupt the p53/MDM2 interaction result in the accumulation of transcriptionally active wild-type p53. It has to be mentioned that although designated as 'non-genotoxic' p53 activators, both RITA and nutlin-3 have been shown to induce DNA damage in the meantime..$^{3-5}$

Recently, we and others have shown that treatment of cancer cells with nutlin-3 induces p53 mutations in p53 wild-type cancer cells from different entities. ${ }^{6,7}$ Moreover, p53 wild-type cancer cell adaptation to nutlin-3 results in a multi-drug resistance phenotype and decreased cellular sensitivity to irradiation. ${ }^{7,8}$ Repeated adaptation of a single p53 wild-type cell-derived clone of the neuroblastoma cell line UKF-NB-3 to nutlin-3 resulted in the

\footnotetext{
${ }^{1}$ Institut für Medizinische Virologie, Klinikum der Goethe-Universität, Paul Ehrlich-Str. 40, 60596 Frankfurt am Main, Germany; ${ }^{2}$ blue-drugs GmbH, Komturstr. 3 A, 60528 Frankfurt am Main, Germany; ${ }^{3}$ Department of Neuropathology, Ruprecht-Karls-University Heidelberg, Im Neuenheimer Feld 220/221, 69120 Heidelberg, Germany; ${ }^{4}$ Deutsches Krebsforschungszentrum, Im Neuenheimer Feld 280, 69120 Heidelberg, Germany; Institute of Molecular, Cell and Systems Biology, College of Medical, Veterinary and Life Sciences, Joseph Black Building, B3.10, University of Glasgow G12 8QQ, Glasgow, Scotland, United Kingdom; ${ }^{6}$ Groningen Bioinformatics Centre, University of Groningen, Nijenborgh 7, $9747 \mathrm{AG}$, Groningen, The Netherlands; ${ }^{7}$ Klinik für Strahlentherapie und Onkologie, Klinikum der Goethe-Universität, Theodor-Stern-Kai 7, 60590 Frankfurt am Main, Germany; ${ }^{8}$ Children's Medical Research Institute, 214 Hawkesbury Road, Westmead, 2145 New South Wales, Australia and ${ }^{9}$ Sydney Medical School, The University of Sydney, Sydney, 2006 New South Wales, Australia

${ }^{*}$ Corresponding author: J Cinatl Jr, Institut für Medizinische Virologie, Klinikum der Goethe-Universität, Paul Ehrlich-Str. 40, 60596 Frankfurt am Main, Germany. Tel: +49 696301 6409; Fax +49696301 4302; E-mail: Cinatl@em.uni-frankfurt.de

${ }^{10}$ Current address: School of Biosciences, University of Kent, Canterbury CT2 7NJ, UK.

Keywords: RITA; nutlin-3; p53; p53 activator; drug resistance; radiation

Abbreviatons: CDDP, cisplatin; DOX, doxorubicin; FDR, false discovery rate; MEL, melphalan; MDM2, murine double minute 2; MTT, 3-(4,5-dimethylthiazol-2-yl)2,5-diphenyltetrazolium bromide; PARP, Poly (ADP-ribose) polymerase; VCR, vincristine

Received 08.2.12; revised 01.3.12; accepted 02.3.12; Edited by A Stephanou
} 
establishment of sub-lines harbouring various p53 mutations, indicating nutlin-3 to induce de novo p53 mutations. ${ }^{7}$

However, it remains unclear whether the induction of p53 mutations and the emergence of multi-drug resistance are a specific feature of nutlin-3 treatment or a common attribute of the whole class of agents that interfere with the interaction of MDM2 and p53.

In this study, we investigated the long-term effects of the non-genotoxic p53 activator RITA that inhibits the p53/MDM2 interaction by binding to p53. We employed the same wildtype p53 expressing neuroblastoma cell line UKF-NB-3 that we have used previously to study adaptation to nutlin-3. ${ }^{7}$ Here, we show that - unlike the MDM2 inhibitor nutlin-3 continuous treatment with RITA does not induce loss-offunction mutations in the TP53 gene. In further contrast to nutlin-3 treatment, cells adapted to RITA displayed no or minor multi-drug resistance phenotypes and a substantially lower degree of resistance to irradiation. Moreover, RITAadapted cells remain responsive to nutlin-3.

\section{Results}

UKF-NB-3 neuroblastoma cells adapted to RITA retain functional p53. UKF-NB-3 cells were adapted to growth in the presence of RITA $10 \mu \mathrm{M}$ in 11 independent experiments. The resulting UKF-NB-3 sub-lines were designated as UKFNB-3 ${ }^{r} R I T A^{10 \mu \mathrm{M}}$ as well as UKF-NB-3 ${ }^{\mathrm{r}} \mathrm{RITA}^{10 \mu \mathrm{M}} \mathrm{I}-\mathrm{X}$ (Table 1). Adaptation was performed by UKF-NB-3 cell cultivation in increasing RITA concentrations as described before for cancer cell adaptation to other drugs. ${ }^{7,9}$ Only one RITAresistant UF-NB-3 sub-line (UKF-NB-3 ${ }^{\mathrm{r}} \mathrm{RITA}{ }^{10 \mu \mathrm{M}} \mathrm{IV}$ ) carried a (heterozygote A76T) p53 mutation (Table 1).

Codon 76 is located in the proline-rich domain of p53, a region that is rarely affected by mutations in cancer. Indeed, only one study has reported an A76T mutation that was found in benign breast tissue (Kandel et al ${ }^{11}$; http://www-p53.iarc.fr/). Interestingly, UKF-NB-3 ${ }^{r}$ RITA ${ }^{10 \mu} \mathrm{M} I V$ cells were as sensitive to nutlin-3 treatment as parental UKF-NB-3 cells (Table 1) with nutlin-3 treatment resulting in the induction of $p 53$ response gene expression in these cells (Figure 1). In accord with a study in yeast, ${ }^{11}$ this suggests that the observed A76T mutation has no or only minimal impact on the activity of p53. Collectively, these data demonstrate that adaptation to RITA does not promote inactivation of p53 by mutation. In strong contrast, we have shown recently that adaptation to nutlin-3 in the same cell line has resulted in inactivating mutations in 13 out of 20 sub-lines. ${ }^{7}$

Previously, we have shown that the G245C p53-mutated nutlin-3-adapted UKF-NB-3 sub-line UKF-NB-3'Nutlin ${ }^{10 \mu M}$

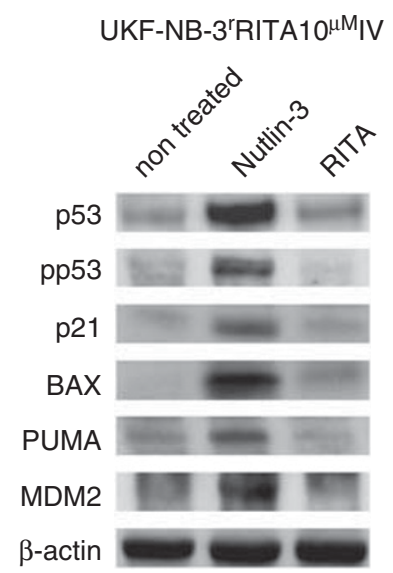

Figure 1 p53 activation in A76T p53-mutated UKF-NB-3 ${ }^{r}$ RITA ${ }^{10 \mu \mathrm{M}} \mathrm{IV}$ cells. Cells were treated with RITA $(10 \mu \mathrm{M})$ or nutlin- $3(10 \mu \mathrm{M})$ for $24 \mathrm{~h}$ and investigated by western blot for the expression of p53 target genes

Table 1 Sensitivity of RITA- and nutlin-3-adapted UKF-NB-3 sub-lines to anticancer drugs

\begin{tabular}{|c|c|c|c|c|c|}
\hline \multirow[b]{2}{*}{ Cell line } & \multirow[b]{2}{*}{ p53 status } & \multicolumn{4}{|c|}{ Concentrations that decrease cell viability by $50 \%\left(\mathrm{IC}_{50}\right)$} \\
\hline & & RITA $(\mu \mathrm{M})$ & Nutlin-3 ( $\mu \mathrm{M})$ & Vincristine (ng/ml) & Cisplatin (ng/ml) \\
\hline 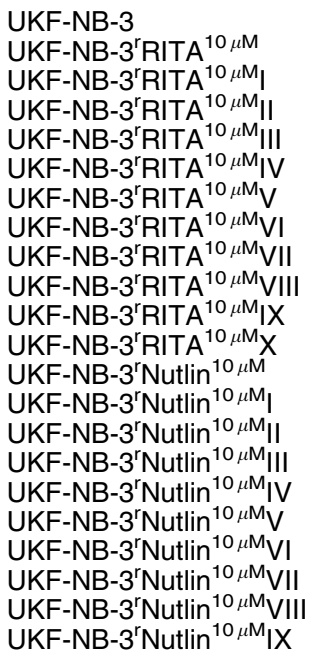 & $\begin{array}{c}w^{\mathrm{a}} \\
w t \\
w t \\
w t \\
w t \\
\text { A76T } \\
\text { wt } \\
w t \\
w t \\
w t \\
w t \\
w t \\
\text { G245C } \\
w t \\
w t \\
w t \\
w t \\
w t \\
P 278 A^{c} \\
D 281 G^{c} \\
D 281 G^{c} \\
D 281 G^{c}\end{array}$ & $\begin{array}{r}0.10 \pm 0.03 \\
12.57 \pm 1.25 \\
8.32 \pm 1.32 \\
5.99 \pm 1.80 \\
10.79 \pm 0.70 \\
6.20 \pm 2.37 \\
9.61 \pm 1.96 \\
11.00 \pm 2.94 \\
9.05 \pm 2.09 \\
6.18 \pm 0.16 \\
13.63 \pm 2.54 \\
7.93 \pm 1.68 \\
0.59 \pm 0.19 \\
0.15 \pm 0.03 \\
0.50 \pm 0.14 \\
0.15 \pm 0.05 \\
0.17 \pm 0.03 \\
0.12 \pm 0.04 \\
0.10 \pm 0.02 \\
0.40 \pm 0.05 \\
0.63 \pm 0.20 \\
0.48 \pm 0.12\end{array}$ & $\begin{array}{l}1.53 \pm 0.30 \\
0.97 \pm 0.33 \\
1.05 \pm 0.08 \\
1.42 \pm 0.10 \\
1.89 \pm 0.34 \\
1.62 \pm 0.07 \\
1.76 \pm 0.06 \\
1.36 \pm 0.10 \\
1.77 \pm 0.14 \\
1.28 \pm 0.13 \\
1.88 \pm 0.28 \\
2.01 \pm 0.47 \\
36.7 \pm 2.9 \\
34.0 \pm 4.8 \\
40.0 \pm 14.0 \\
35.2 \pm 10.1 \\
33.6 \pm 9.9 \\
34.3 \pm 11.6 \\
35.3 \pm 14.2 \\
40.5 \pm 13.9 \\
40.9 \pm 4.5 \\
47.2 \pm 5.1\end{array}$ & $\begin{array}{l}0.20 \pm 0.04 \\
0.32 \pm 0.09 \\
0.23 \pm 0.08 \\
0.25 \pm 0.06 \\
0.40 \pm 0.10 \\
0.22 \pm 0.08 \\
0.22 \pm 0.05 \\
0.31 \pm 0.07 \\
0.27 \pm 0.06 \\
0.25 \pm 0.03 \\
0.29 \pm 0.06 \\
0.34 \pm 0.05 \\
1.27 \pm 0.20 \\
0.70 \pm 0.09 \\
1.00 \pm 0.21 \\
0.59 \pm 0.03 \\
0.57 \pm 0.05 \\
0.72 \pm 0.07 \\
1.07 \pm 0.11 \\
0.62 \pm 0.02 \\
1.07 \pm 0.18 \\
0.95 \pm 0.15\end{array}$ & $\begin{array}{c}99.8 \pm 24.1 \\
177.1 \pm 41.7 \\
73.4 \pm 14.0 \\
116.3 \pm 17.6 \\
159.3 \pm 36.8 \\
98.1 \pm 6.1 \\
106.9 \pm 24.7 \\
114.4 \pm 21.8 \\
154.0 \pm 20.1 \\
107.4 \pm 12.9 \\
103.4 \pm 5.4 \\
124.8 \pm 30.8 \\
303.1 \pm 14.3 \\
191.7 \pm 7.5 \\
150.5 \pm 14.5 \\
150.5 \pm 14.5 \\
145.5 \pm 15.9 \\
112.2 \pm 9.7 \\
150.0 \pm 13.3 \\
180.9 \pm 39.6 \\
182.6 \pm 30.6 \\
312.6 \pm 32.8\end{array}$ \\
\hline
\end{tabular}

aWild-type. ${ }^{\text {b}}$ Type of mutation. ${ }^{\mathrm{C}}$ Heterozygote. ${ }^{\mathrm{d}}$ Homozygote 
retains its resistance phenotype after multiple passages in the absence of nutlin-3. ${ }^{7}$ Here, we cultivated the investigated RITA-adapted UKF-NB-3 sub-lines for 10 passages in the absence of RITA. As a control, we also cultivated 10 nutlin-3adapted UKF-NB-3 sub-lines (five p53-mutated, five p53 wild-type) for 10 passages without adding nutlin-3. All of the nutlin-3-adapted cell lines remained nutlin-3 resistant, independently of their p53 status. In contrast, 2 out of the 11 RITA-adapted cell lines (UKF-NB-3 ${ }^{r}$ RITA ${ }^{10 \mu \mathrm{M}}$ III, UKF-NB$3^{r} R^{\prime} A^{10 \mu M}$ VII) retrieved their RITA sensitivity (Supplementary Table 1).

RITA-adapted cells remain sensitive to nutlin-3. Next, we determined cross-resistance profiles between the RITAadapted UKF-NB-3 sub-lines and the nutlin-3-adapted UKF-NB-3 sub-lines (Table 1). The RITA concentrations that decreased the viabilities of the RITA-adapted cell lines by $50 \%\left(\mathrm{IC}_{50}\right)$ were about 60-120-fold higher than the RITA $\mathrm{IC}_{50}$ in UKF-NB-3 cells $(0.10 \mu \mathrm{M})$. None of the RITA-adapted sub-lines (including the A76T p53-mutated sub-line UKF-NB$\left.3^{r} R_{T} A^{10 \mu M} I V\right)$ showed cross-resistance to nutlin-3. Moreover, when the RITA-adapted UKF-NB-3 cell line UKF-NB$3^{r} R_{T T A}{ }^{10 \mu M}$ was adapted to nutlin-3 in six independent experiments, we found p53 mutations in four sub-lines whereas only two sub-lines retained a wild-type p53 sequence (Supplementary Table 2). One nutlin-3-adapted sub-line of UKF-NB-3r $3^{r}$ ITA ${ }^{10 \mu \mathrm{M}}$ harboured a heterozygote N239D mutation, one a heterozygote R280I mutation, and two harboured heterozygote C277F mutations. These results demonstrate that, even after adaptation to the p53 activator RITA, exposure to the MDM2 inhibitor nutlin-3 exerts a strong selection pressure favouring p53 mutations.

Four out of five p53-mutated nutlin-3-adapted UKF-NB-3 sub-lines displayed decreased sensitivity to RITA, supporting the importance of wild-type p53 for RITA-induced toxicity. $I_{50}$ values in these sub-lines ranged from 0.40 to $0.63 \mu \mathrm{M}$ (compared with $0.10 \mu \mathrm{M}$ in the parental UKF-NB-3), whereas only one p53-mutated sub-line (UKF-NB-3r Nutlin ${ }^{10 \mu \mathrm{M}} \mathrm{VI}$ ) was as sensitive to RITA as the parental cells. Consistently, all but one (UKF-NB-3 ${ }^{r}$ Nutlin $^{10 \mu \mathrm{M}} \mathrm{II}$ ) of the nutlin-3-adapted sub-lines expressing wild-type p53 were as sensitive to RITA as the parental cells (Table 1).

RITA-adapted cells display a less pronounced resistance phenotype than nutlin-3-adapted cells. Adaptation of UKF-NB-3 cells to nutlin-3 results in decreased sensitivity to a panel of different cytotoxic drugs and to irradiation as determined in the p53 (G245C)-mutated sub-line UKF-NB$3^{r}$ Nutlin $^{10 \mu \mathrm{M}}$. To test whether adaptation to RITA is associated with a similar multi-drug resistance phenotype, we compared the sensitivities of the 11 RITA-adapted UKF-NB-3 sub-lines and 10 additional nutlin-3-adapted UKF-NB-3 sub-lines to vincristine (VCR) and cisplatin (CDDP; Table 1). Only two out of the 11 RITA-adapted cell lines displayed significantly increased $(P<0.05)$ VCR $\mathrm{IC}_{50}$ values (UKF-NB-3 ${ }^{\mathrm{r}} \mathrm{RITA}{ }^{10 \mu \mathrm{M}} \mathrm{III}, 0.40 \mathrm{ng} / \mathrm{ml}$; UKF-NB$3^{\mathrm{r}} \mathrm{RITA}{ }^{10 \mu \mathrm{M}} \mathrm{X}, 0.34 \mathrm{ng} / \mathrm{ml}$ ) relative to parental UKF-NB-3 cells $(0.20 \mathrm{ng} / \mathrm{ml})$, whereas the majority of RITA-adapted cell lines remained VCR sensitive. In contrast, all of the 10 nutlin-3adapted UKF-NB-3 cell lines showed significantly enhanced
VCR $\mathrm{IC}_{50}$ values ranging from 0.57 to $1.27 \mathrm{ng} / \mathrm{ml}$. Similar results were obtained for CDDP: three RITA-adapted sublines were significantly less sensitive to $\operatorname{CDDP}(P<0.05$; $I_{50}$ S: UKF-NB-3 ${ }^{\mathrm{r}} \mathrm{RITA}^{10 \mu \mathrm{M}}, 177.1 \mathrm{ng} / \mathrm{ml}$; UKF-NB-3 ${ }^{\mathrm{r}} \mathrm{RITA}^{10 \mu \mathrm{M}} \mathrm{III}$, $159.3 \mathrm{ng} / \mathrm{ml}$; UKF-NB-3 $\left.{ }^{\mathrm{r}} \mathrm{RITA}{ }^{10 \mu \mathrm{M}} \mathrm{VII}, 154.0 \mathrm{ng} / \mathrm{ml}\right)$ than the UKF-NB-3 cells $(99.8 \mathrm{ng} / \mathrm{ml})$, whereas all nutlin-3-adapted sub-lines showed significantly enhanced $I_{50}$ values ranging from 145.5 to $312.6 \mathrm{ng} / \mathrm{ml}$ with the exception of UKF-NB$3^{\mathrm{r} N u t l i n}{ }^{10 \mu \mathrm{M}} \mathrm{V}(112.2 \mathrm{ng} / \mathrm{ml})$ (Table 1$)$.

Interestingly, the multi-drug resistance phenotype observed in the nutlin-3-adapted UKF-NB-3 sub-lines appears more similar to the multi-drug resistance phenotypes observed in cell lines adapted to cytotoxic drugs than the low-level crossresistance patterns observed in the RITA-adapted sub-lines. Investigation of the sensitivity of a panel of UKF-NB-3 sublines adapted to the cytotoxic agents CDDP, doxorubicin (DOX), melphalan (MEL), or VCR displayed generally decreased sensitivity to CDDP and VCR (Supplementary Table 3).

RITA-adapted cells display lower irradiation resistance than nutlin-3-adapted cells. Previously, we showed that the nutlin-3-adapted sub-line UKF-NB-3 ${ }^{\mathrm{r}}$ Nutlin $^{10 \mu \mathrm{M}}$ is highly resistant to irradiation when compared with the parental UKF-NB-3 cell line. ${ }^{7}$ Here, the investigated RITA- and nutlin3-adapted cell lines were irradiated with 3, 5, or $8 \mathrm{~Gy}$, respectively, and cell viability was determined by the 3-(4,5dimethylthiazol-2-yl)-2,5-diphenyltetrazolium bromide (MTT) assay $48 \mathrm{~h}$ post-irradiation. Across all doses, the sensitivity to irradiation was substantially lowered in all cell lines adapted to nutlin-3 when compared with the parental UKF-NB-3 cells (Table 2). For example, the cell viability (expressed relative to non-irradiated control) ranged from $64.64 \pm 2.36 \%$ (UKFNB-3 ${ }^{\mathrm{r}} \mathrm{Nutlin}^{10 \mu \mathrm{M}} \mathrm{V}$ ) to $86.73 \pm 4.22 \%$ (UKF-NB-3 ${ }^{\mathrm{r}}$ Nutlin $^{10 \mu \mathrm{M}} \mathrm{III}$ ) after $5 \mathrm{~Gy}$ irradiation whereas it was $0.36 \pm 0.26 \%$ for UKF-NB-3 cells. In this regard, nutlin-3-adapted cells again behaved similar to UKF-NB-3 sub-lines adapted to genotoxic agents (CDDP, DOX, and VCR) that also showed significant resistance to radiation (Supplementary Table 4). Of note, there was no correlation between the p53 status (wild type or mutant) and irradiation sensitivity in the nutlin-3-adapted cell lines (Table 2).

In contrast, RITA-adapted cells showed only moderate resistance to irradiation with the observed effects being significantly less profound than in the nutlin-3- or cytotoxic drug-adapted cells. After $5 \mathrm{~Gy}$ irradiation, viabilities ranged from $3.89 \pm 2.30 \%$ (UKF-NB-3 $\left.{ }^{r} R^{2} I^{10 \mu \mathrm{M}} I X\right)$ to $38.16 \pm 4.37 \%$ (UKF-NB-3r RITA ${ }^{10 \mu \mathrm{M}}$ II) $($ Table 2).

RITA induces p53 activation and apoptosis in UKF-NB-3 cells. The effects of RITA on p53 activation and apoptosis were studied in UKF-NB-3 cells, the RITA-adapted sub-line UKF-NB-3 ${ }^{\mathrm{r}} \mathrm{RITA}{ }^{10 \mu \mathrm{M}}$ (harbouring wild-type p53), and the nutlin-3-adapted sub-line UKF-NB-3 ${ }^{r}$ Nutlin $^{10 \mu M}$ (harbouring G245C mutant p53) upon treatment with RITA and nutlin-3, respectively (Figure 2). In the parental cells, RITA $10 \mu \mathrm{M}$ as well as nutlin-3 $10 \mu \mathrm{M}$ induced accumulation and phosphorylation of p53 at serine 15, which both are hallmarks of p53 activation. In addition, both drugs increased expression of the p53 target genes CDKNA1 (encoding for p21), BAX, 
Table 2 Sensitivity of RITA- and nutlin-3-adapted UKF-NB-3 sub-lines to irradiation. Cell viability was determined $48 \mathrm{~h}$ post-irradiation by MTT assay

\begin{tabular}{|c|c|c|c|c|}
\hline \multirow[b]{2}{*}{ Cell line } & \multirow[b]{2}{*}{ p53 status } & \multicolumn{3}{|c|}{ Cell viability (\% non-irradiated control) } \\
\hline & & 3 Gy & 5 Gy & 8 Gy \\
\hline 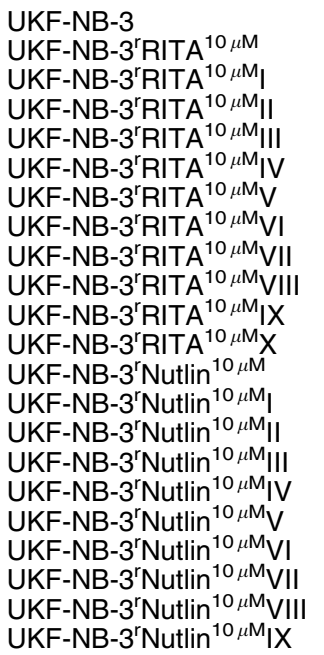 & $\begin{array}{c}w^{\mathrm{a}} \\
w t \\
w t \\
w t \\
w t \\
\text { A76T } \\
\text { wt } \\
\text { wt } \\
\text { wt } \\
\text { wt } \\
\text { wt } \\
\text { wt } \\
\text { G245C } \\
\text { wt } \\
\text { wt } \\
\text { wt } \\
\text { wt } \\
\text { wt } \\
\text { P278A } \\
\text { D281G } \\
\text { D281G } \\
\text { D281G }\end{array}$ & $\begin{array}{c}7.79 \pm 3.54 \\
48.07 \pm 5.21 \\
22.30 \pm 2.37 \\
45.98 \pm 5.78 \\
34.64 \pm 2.69 \\
16.13 \pm 2.76 \\
37.52 \pm 3.39 \\
32.36 \pm 5.36 \\
25.03 \pm 5.45 \\
26.40 \pm 6.20 \\
14.39 \pm 2.75 \\
41.97 \pm 4.55 \\
89.44 \pm 5.08 \\
74.19 \pm 3.41 \\
81.77 \pm 15.00 \\
86.79 \pm 3.63 \\
88.40 \pm 1.93 \\
72.45 \pm 10.85 \\
81.68 \pm 8.58 \\
77.86 \pm 11.98 \\
84.25 \pm 8.96 \\
83.46 \pm 10.63\end{array}$ & $\begin{array}{c}0.36 \pm 0.26 \\
25.90 \pm 2.25 \\
14.70 \pm 2.47 \\
38.16 \pm 4.37 \\
26.52 \pm 3.29 \\
9.40 \pm 1.02 \\
23.17 \pm 1.63 \\
14.66 \pm 1.46 \\
16.23 \pm 1.20 \\
20.67 \pm 1.99 \\
3.89 \pm 2.30 \\
21.95 \pm 4.17 \\
85.69 \pm 8.10 \\
71.28 \pm 4.36 \\
74.44 \pm 10.03 \\
86.73 \pm 4.22 \\
85.60 \pm 3.49 \\
64.64 \pm 2.36 \\
74.23 \pm 2.14 \\
69.04 \pm 10.54 \\
67.99 \pm 4.88 \\
83.51 \pm 4.42\end{array}$ & $\begin{array}{c}0 \\
13.91 \pm 2.36 \\
9.02 \pm 1.68 \\
12.73 \pm 2.52 \\
9.38 \pm 1.56 \\
5.45 \pm 1.73 \\
7.22 \pm 1.34 \\
12.89 \pm 1.38 \\
10.71 \pm 2.18 \\
16.82 \pm 1.94 \\
0 \\
3.32 \pm 0.49 \\
68.92 \pm 3.54 \\
59.28 \pm 8.74 \\
70.12 \pm 19.47 \\
68.21 \pm 18.25 \\
60.05 \pm 14.16 \\
55.63 \pm 8.64 \\
72.33 \pm 8.98 \\
42.03 \pm 7.64 \\
64.47 \pm 6.17 \\
44.32 \pm 8.18\end{array}$ \\
\hline
\end{tabular}

aWild-type. ${ }^{\mathrm{b}}$ Type of mutation. ${ }^{\mathrm{c}}$ Heterozygote. ${ }^{\mathrm{d}}$ Homozygote

BBC3 (encoding for PUMA), and MDM2 with nutlin-3 clearly exerting more pronounced effects. These results confirm that, as expected, both drugs activate wild-type p53. In line with their p53 status (mutated) neither nutlin-3 nor RITA induced a p53 response in UKF-NB-3 $3^{r}$ Nutlin $^{10 \mu M}$ cells. However, nutlin-3 treatment resulted in a robust p53 response in UKF-NB-3 ${ }^{\mathrm{r}} \mathrm{RITA} \mathrm{A}^{10 \mu \mathrm{M}}$ cells (Figure $2 \mathrm{a}$ ) indicating that adaptation to RITA does not abolish the functionality of p53. These data show that RITA-adapted cells although resistant to the p53 activator RITA remain sensitive to p53 activation by the MDM2 inhibitor nutlin-3.

Both substances induced apoptosis in UKF-NB-3 cells as indicated by Poly (ADP-ribose) polymerase (PARP) cleavage (Figure 2b) and caspase 3 activation (Figure 2d). Consistent to the $\mathrm{IC}_{50}$ values measured before (Table 1) RITA retained its ability to elicit apoptosis in UKF-NB-3 ${ }^{r}$ Nutlin $^{10 \mu \mathrm{M}}$ cells. Similarly, nutlin-3 caused apoptosis in UKF-NB-3 ${ }^{r}$ RITA $^{10 \mu \mathrm{M}}$ cells (Figures $2 b$ and $c$ ).

To corroborate the importance of wild-type p53 for apoptosis induced by nutlin-3, we had previously shown that transduction of UKF-NB-3 ${ }^{r}$ Nutlin ${ }^{10 \mu \mathrm{M}}$ cells with a lentiviral vector encoding for wild-type p53 (UKF-NB-3 ${ }^{\mathrm{r}}$ Nutlin $^{10 \mu \mathrm{Mwtp} 53}$ ) resulted in their re-sensitisation to nutlin-3. ${ }^{7}$ Similarly,

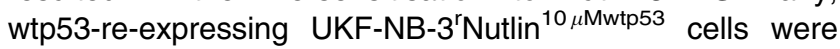
significantly more sensitive to RITA when compared with

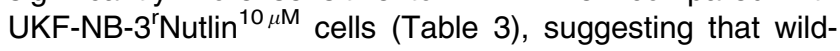
type p53 function accounts for the major part of RITA-induced cytotoxicity. Collectively, these experiments demonstrate that RITA and nutlin-3 both elicit apoptosis via p53.

To characterise the differences underlying the different phenotypes of UKF-NB-3 cells adapted to RITA and nutlin-3, respectively, we looked at the expression level of selected antiapoptotic factors. UKF-NB- $3^{r}$ RITA ${ }^{10 \mu M}$ cells showed an enhanced expression of the antiapoptotic proteins CIAP1 and
clAP2 whereas UKF-NB-3 ${ }^{r}$ Nutlin $^{10 \mu} \mathrm{M}$ cells expressed increased levels of the antiapoptotic proteins cIAP1, Bcl-2, $\mathrm{Mcl}-1$, and survivin compared with UKF-NB-3 (Figure 3).

Transcriptomics analysis indicates a closer relationship between UKF-NB-3 cells and RITA-adapted cells than between UKF-NB-3 and nutlin-3-adapted cells. Next, we analysed global cellular gene expression at the mRNA level using gene microarray in UKF-NB-3, UKF-NB-3r Nutlin ${ }^{10 \mu \mathrm{M}} \mathrm{Il}$, UKF-NB-3 ${ }^{r}$ Nutlin ${ }^{10 \mu \mathrm{M}} \mathrm{V}$, UKF-NB-3 ${ }^{\mathrm{r}}$ Nutlin ${ }^{10 \mu \mathrm{M}} \mathrm{VI}$, UKF-NB$3^{r}$ Nutlin $^{10 \mu \mathrm{M}}$ VIII, UKF-NB-3r RITA ${ }^{10 \mu \mathrm{M}} \mathrm{II}$, UKF-NB-3r RITA ${ }^{10 \mu \mathrm{M}} \mathrm{III}$, and UKF-NB-3 $3^{r}$ RITA ${ }^{10 \mu \mathrm{M}} \mathrm{IV}$ cells, using three biological replicates for each cell line. In general, nutlin-3-adapted cells showed a larger number of statistically significant gene expression changes compared with the parental line than the RITA-adapted cells (Table 4). The numbers of differentially expressed genes were 6465 for the comparison UKF-NB-3 versus nutlin-3-adapted sub-lines and 1495 for the comparison UKF-NB-3 versus RITA-adapted sub-lines (false discovery rate (FDR) $<0.05$ after correction for multiple testing). Also in the individual comparisons of the drug-adapted cell lines versus UKF-NB-3, the nutlin-3-adapted cell lines showed generally higher numbers of differentially expressed genes (4831-6575) compared with the RITA-adapted lines (1985-3606). The only exception was UKF-NB-3 ${ }^{\mathrm{r}} \mathrm{Nutlin}^{10 \mu \mathrm{M}} \mathrm{II}$ (2528 differentially expressed genes; Table 4).

In a hierarchical clustering analysis, the parental UKF-NB-3 cell line clustered together with the RITA-adapted cell lines whereas the nutlin-3-adapted cells formed a separate cluster (Figure 4). Within the UKF-NB-3 and RITA-adapted cell cluster, UKF-NB-3 clustered together with UKF-NB-3 ${ }^{r}$ RITA ${ }^{10 \mu \mathrm{M}} I$, the cell line with the lowest number of genes differentially expressed to UKF-NB-3. Looking at the correlation distances, the nutlin-3-adapted cells appear to be slightly more 


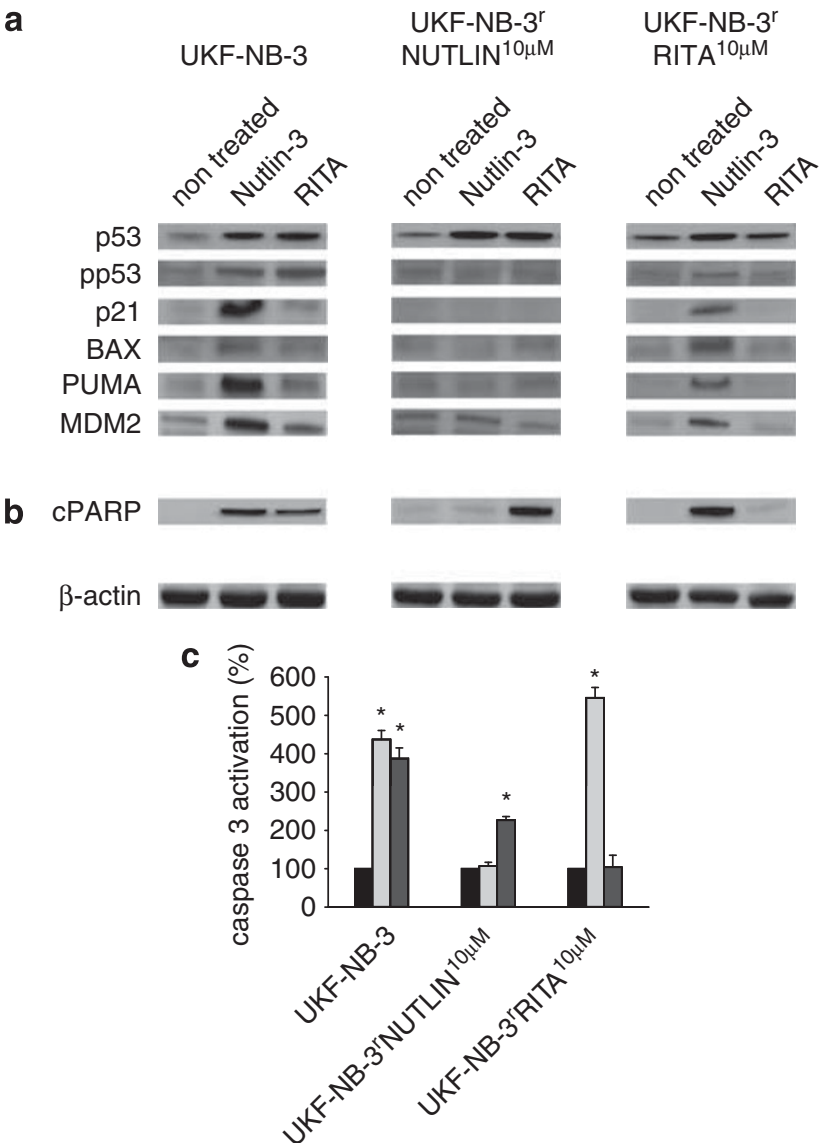

Figure 2 Influence of RITA and nutlin-3 on p53 activation and apoptosis in UKF-NB-3, UKF-NB-3'Nutlin ${ }^{10 \mu \mathrm{M}}$, and UKF-NB-3 ${ }^{\mathrm{R}}$ RITA ${ }^{10 \mu \mathrm{M}}$ cells. (a-b) Cells were treated with RITA $(10 \mu \mathrm{M})$ or Nutlin-3 $(10 \mu \mathrm{M})$ for $24 \mathrm{~h}$ and investigated by western blot for the expression of p53 target genes (a) or for the formation of the cleaved form of PARP (CPARP) (b). $\beta$-actin served as loading control. (c) Cells were treated with RITA $(10 \mu \mathrm{M})$ or Nutlin-3 $(10 \mu \mathrm{M})$ for $24 \mathrm{~h}$. Caspase 3 activation was determined by Caspase-Glo 3/7 Assay. ${ }^{*} P<0.05$

Table 3 Sensitivity of UKF-NB-3 cells, UKF-NB- $3^{r}$ Nutlin $^{10 \mu \mathrm{M}}$ cells, UKF-NB$3^{r} \mathrm{Nutlin}^{10 \mu \mathrm{M}}$ cells transduced with a lentiviral vector encoding wild-type p53 (UKF-NB-3 ${ }^{r}$ Nutlin ${ }^{10 \mu \mathrm{Mwtp} 53}$ ), and UKF-NB-3 ${ }^{\mathrm{r}} \mathrm{Nutlin}^{10 \mu \mathrm{M}}$ cells transduced with a control vector (UKF-NB-3 ${ }^{r}$ Nutlin $^{10} \mu$ Mcontrol) ) to RITA or nutlin-3 indicated by the concentration that reduces cell viability by $50 \%\left(\mathrm{IC}_{50}\right)$ indicated by MTT assay after a 5 day treatment period

\begin{tabular}{lcc}
\hline Cell line & RITA $(\boldsymbol{\mu M})$ & Nutlin-3 $(\boldsymbol{\mu M})$ \\
\hline UKF-NB-3 & $0.11 \pm 0.02$ & $1.47 \pm 0.15$ \\
UKF-NB-3rNutlin ${ }^{10 \mu \mathrm{M}}$ & $0.62 \pm 0.04$ & $32.83 \pm 2.91$ \\
UKF-NB-3rNutlin $^{10 \mu \text { Mwtp53 }}$ & $0.24 \pm 0.03$ & $5.02 \pm 1.73$ \\
UKF-NB-3rNutlin $^{10 \mu \text { Mcontrol }}$ & $0.58 \pm 0.03$ & $36.19 \pm 3.08$ \\
\hline
\end{tabular}

homogeneous than the RITA-adapted cells although the nutlin-3-adapted cells consist of two cell lines displaying loss-of-function p53 mutations (UKF-NB-3 $3^{\mathrm{r}} \mathrm{Nutlin}^{10 \mu \mathrm{M}} \mathrm{VI}$, UKFNB-3 $\left.3^{r} \mathrm{Nutlin}^{10 \mu \mathrm{M}} \mathrm{VIII}\right)$ and two that harbour wild-type p53 (UKFNB-3rNutlin ${ }^{10 \mu \mathrm{M}} \mathrm{II}$, UKF-NB-3 $3^{r}$ Nutlin $^{10 \mu \mathrm{M}} \mathrm{V}$ ) whereas all three RITA-adapted cell lines harbour functional p53. Within the cluster containing the nutlin-3-adapted cell line,

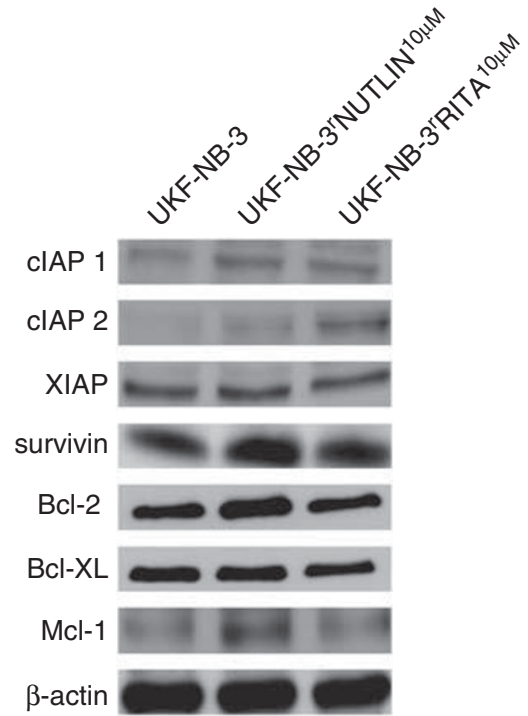

Figure 3 Expression of apoptosis-associated proteins in UKF-NB-3, UKF-NB$3^{r}$ Nutlin $^{10 \mu \mathrm{M}}$, and UKF-NB-3'RITA ${ }^{10 \mu \mathrm{M}}$ cells

Table 4 Numbers of genes differentially expressed relative to UKF-NB-3 (false discovery rate (FDR) $<0.05$ or $<0.01$ after correction for multiple testing) in RITA- or nutlin-3-adapted UKF-NB-3 sub-lines as determined by microarray measurement of mRNA abundance

\begin{tabular}{|c|c|c|}
\hline $\begin{array}{l}\text { Comparison } \\
\text { UKF-NB-3 versus }\end{array}$ & $\begin{array}{c}\text { Differentially } \\
\text { expressed } \\
\text { genes } \\
(\text { FDR }<0.05)\end{array}$ & $\begin{array}{c}\text { Differentially } \\
\text { expressed } \\
\text { genes } \\
(\text { FDR }<0.01)\end{array}$ \\
\hline All sub-lines & 1360 & 520 \\
\hline All RITA-resistant sub-lines & 1495 & 676 \\
\hline All nutlin-3-resistant sub-lines & 6465 & 4090 \\
\hline UKF-NB-3 ${ }^{r} R_{T} A^{10 \mu \mathrm{M}}$ II & 1985 & 374 \\
\hline UKF-NB-3 ${ }^{\mathrm{r}} \mathrm{RITA}{ }^{10 \mu \mathrm{M}_{I I I}}$ & 2465 & 337 \\
\hline UKF-NB-3 ${ }^{r} R^{2} A^{10 \mu \mathrm{M} I V}$ & 3606 & 817 \\
\hline UKF-NB-3' ${ }^{r}$ Nutlin ${ }^{10 \mu \mathrm{M}_{I I}}$ & 2528 & 75 \\
\hline UKF-NB-3 ${ }^{\mathrm{r}}$ Nutlin $^{10 \mu \mathrm{M}} \mathrm{V}$ & 6575 & 2842 \\
\hline UKF-NB-3'Nutlin ${ }^{10 \mu \mathrm{M}}$ VI & 4831 & 1148 \\
\hline UKF-NB-3' Nutlin ${ }^{10 \mu \mathrm{M}}$ VIII & 5830 & 2004 \\
\hline
\end{tabular}

p53 wild-type cells cluster together with p53-mutated cells. UKF-NB-3 ${ }^{r}$ Nutlin ${ }^{10 \mu \mathrm{M}}$ II (wild-type p53) clusters together with UKF-NB-3 ${ }^{r} \mathrm{Nutlin}^{10 \mu \mathrm{M} \mathrm{VIII}}$ (p53-mutated), UKF-NB-3 ${ }^{\mathrm{r}}$ Nutlin ${ }^{10 \mu \mathrm{M}} \mathrm{V}$ (wild-type p53) together with UKF-NB-3 ${ }^{r}$ Nutlin ${ }^{10 \mu \mathrm{M}} \mathrm{VI}$ (p53-mutated).

5363 genes were found to be differentially expressed $(F D R<0.05)$ in at least one comparison of UKF-NB-3 versus RITA-adapted UKF-NB-3 cell line. In all, 2030 genes were differentially expressed between UKF-NB-3 and two RITA-adapted cell lines, and 663 genes between UKF-NB-3 and all three investigated RITA-adapted cell lines. A total of 8891 genes were differentially regulated between UKF-NB-3 and at least one nutlin-3-adapted UKF-NB-3 cell line, 5685 consistently between UKF-NB-3 and two nutlin-3adapted cell lines, 3592 between UKF-NB-3 and three nutlin3-adapted cell lines, and 1597 genes between UKF-NB-3 and all four investigated nutlin-3-adapted cell lines (Supplementary Table 5). $17.96 \%$ (1597/8891) of the genes differentially expressed between UKF-NB-3 and any of its 


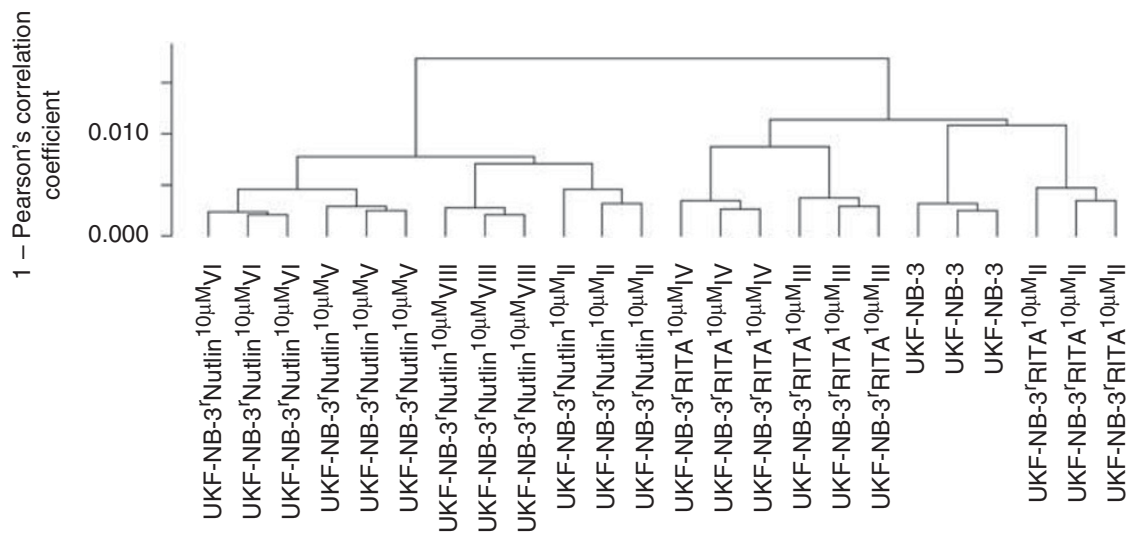

Figure 4 Hierarchical cluster analysis based on transcriptomics data of UKF-NB-3 and UKF-NB-3 sub-lines adapted to RITA- or nutlin-3. Each cell line was analysed in triplicate

nutlin-3-adapted sub-lines were differentially expressed between UKF-NB-3 and all four investigated nutlin-3-adapted sub-lines whereas $12.36 \%(663 / 5363)$ of the genes differentially expressed between UKF-NB-3 and any of its RITAadapted sub-lines were differentially expressed between UKF-NB-3 and all three investigated RITA-adapted sub-lines.

\section{Discussion}

The MDM2 inhibitor nutlin-3 has been shown to induce p53 mutations in different p53 wild-type cell types. ${ }^{6,7}$ In all, 13 out of 20 nutlin-3-adapted sub-lines of the wild-type p53 neuroblastoma cell line UKF-NB-3 harboured p53 mutations. ${ }^{7}$ Here, we established 11 UKF-NB-3 sub-lines adapted to growth in the presence of RITA, a drug that interferes with the interaction of $\mathrm{p} 53$ and MDM2 by binding to $\mathrm{p} 53 .^{2,12}$ The chemical structures of RITA and nutlin-3 are shown in Supplementary Figure 1. Ten sub-lines harboured wild-type p53, whereas the cell line UKF-NB-3 ${ }^{r}$ RITA ${ }^{10 \mu \mathrm{M}}$ IV harboured an A76T mutation that did not result in a loss of p53 function and remained sensitive to nutlin-3-induced anticancer effects and nutlin-3-induced p53 activation. In accordance, a previous study performed in a yeast system had shown that the transactivation capacities of A76T-mutated p53 do not differ from those of wild-type p53. ${ }^{11}$ Thus, this mutation does not appear to result from a selective pressure favouring a loss of p53 function. These findings reveal a striking difference between two drugs that disrupt the Mdm2/p53 interaction with only nutlin-3 but not RITA promoting inactivating de novo mutations of p53.

A number of different factors may contribute to the observed differences in the potential of nutlin-3 and RITA to induce p53 mutations. RITA induces a p53 response in UKFNB-3 cells but not in the RITA-adapted UKF-NB-3 sub-line UKF-NB-3 ${ }^{r}$ RITA $^{10 \mu \mathrm{M}}$ indicating that development of resistance to RITA resistance involves the establishment of mechanisms that render RITA-resistant cells insensitive to RITA-induced p53 activation. Re-establishment of wildtype p53 expression in the G245C p53-mutated nutlin-3adapted UKF-NB-3 sub-line UKF-NB-3 ${ }^{\mathrm{r}} \mathrm{Nutlin}^{10 \mu \mathrm{M}}$ had been shown previously to re-sensitise this cell line to nutlin-3. ${ }^{7}$ Here, re-establishment of wild-type p53 expression in
UKF-NB-3 ${ }^{r}$ Nutlin ${ }^{10 \mu \mathrm{M}}$ cells also re-sensitised this cell line to RITA further supporting a role of p53 activation in the anticancer activity of RITA in the investigated model.

In comparison to nutlin-3, however, RITA induced a less pronounced $p 53$ response and the selection pressure towards mutant, non-functional forms of p53 might correlate with the extent of p53 activation. Moreover, RITA was shown to reactivate mutated forms of $\mathrm{p} 53 .{ }^{13}$ Although we did not detect p53 reactivation in G245C p53-mutated UKF-NB-3 ${ }^{r}$ Nutlin $^{10 \mu M}$ cells by RITA treatment, RITA may reactivate other mutant p53 forms and not favour their emergence and/or selection. Differences in the effects on other signalling pathways may also contribute to the observed discrepancies in the induction of p53 mutations. Although RITA targets p53 directly, nutlin-3 also interferes with the interaction of MDM2 and other molecules than p53. ${ }^{14,15}$ Only RITA, but not nutlin-3, interfered with the interaction of p53 and 53BP1 in a yeast assay. ${ }^{16}$ Notably, although G245C p53-mutated UKF-NB-3r Nutlin ${ }^{10 \mu \mathrm{M}}$ cells showed some cross-resistance to RITA, higher RITA concentrations induced caspase-dependent apoptosis in this cell line.

In previous studies, nutlin-3-adapted cancer cells consistently displayed a multi-drug-resistance phenotype and decreased sensitivity to irradiation. ${ }^{7,8}$ Here, all 10 investigated p53 wild-type and p53-mutated nutlin-3-adapted cell lines showed substantially decreased sensitivity to VCR and irradiation. In all, 9 out of 10 nutlin-3-adapted UKF-NB-3 sub-lines were also less sensitive to CDDP treatment than UKF-NB-3. In contrast, only 2 out of 11 RITA-adapted cell lines displayed a reduced sensitivity to VCR (although to a lesser extent than the nutlin-3-adapted cell lines) and only 3 to CDDP. Although the RITA-adapted UKF-NB-3 sub-lines were less sensitive to irradiation than UKF-NB-3, their radiation sensitivity remained substantially higher than that of the nutlin-3-adapted cell lines. In general, the multi-drug resistance phenotype observed in nutlin-3-adapted cells appears to be more similar to the resistance phenotype observed in cytotoxic drug-adapted cells than the low-level resistance found in RITA-adapted cells. Moreover, 2 out of 11 RITA UKFNB-3 sub-lines regained sensitivity to RITA after cultivation for 10 passages in the absence of RITA, whereas all nutlin-3resistant sub-lines remained nutlin-3 resistant. 
Changes in the expression of apoptosis-related proteins could contribute to the generally enhanced resistance phenotype of nutlin-3-adapted UKF-NB-3 cells. In UKF-NB$3^{r}$ Nutlin $^{10 \mu \mathrm{M}}$ cells, multiple antiapoptotic changes were detected. UKF-NB-3 ${ }^{\mathrm{r}}$ Nutlin ${ }^{10 \mu \mathrm{M}}$ cells were characterised by (in comparison to UKF-NB-3 and UKF-NB-3 ${ }^{\mathrm{r}} \mathrm{RITA}^{10 \mu \mathrm{M}}$ ) higher levels of the antiapoptotic proteins clAP1, Bcl-2, Mcl-1, and survivin. In contrast, across the pro- and antiapoptotic proteins tested, UKF-NB-3 ${ }^{r}$ RITA ${ }^{10 \mu \mathrm{M}}$ cells exhibited only enhanced expression of cIAP1 and clAP2 in comparison to UKF-NB-3.

Transcriptomics analyses showed that the RITA-adapted sub-lines are more closely related to the parental UKF-NB-3 cell line than the nutlin-3-adapted cell lines. This also appears to correlate with the observed sensitivity to chemotherapeutic agents and irradiation. The RITA-adapted cells resemble more closely the sensitive UKF-NB-3 phenotype than the multi-drug resistance phenotype associated with nutlin-3 adaptation.

Finally, all RITA-adapted cell lines remained nutlin-3sensitive. Accordingly, nutlin-3 induced a substantial p53 response and caused caspase activation and apoptosis in UKF-NB-3r ${ }^{r}$ ITA ${ }^{10 \mu \mathrm{M}}$ cells. Moreover, four out of six nutlin-3adapted sub-lines of the RITA-adapted p53 wild-type cell line UKF-NB-3 ${ }^{r}$ RITA $^{10 \mu \mathrm{M}}$ harboured p53 mutations. However, five out of ten nutlin-3-adapted UKF-NB-3 sub-lines exhibited decreased sensitivity to RITA treatment. p53 mutations appear to favour RITA resistance as four out of five nutlin-3adapted cell lines that were less sensitive to RITA harboured p53 mutations.

In conclusion, our study reveals a striking difference between the two investigated p53 activators nulin-3 (i.e., under clinical investigation for the treatment of solid tumours and leukaemia in phase I trials ${ }^{2}$ ) and RITA (i.e., under preclinical investigation as anticancer $\left.\operatorname{drug}^{2}\right)$. Whereas adaptation of wild-type p53 expressing UKF-NB-3 cells to nutlin-3 resulted in the emergence of $p 53$ mutations, UKF-NB3 cells adapted to RITA retained functional p53. Consistently, the RITA-adapted cell lines stayed sensitive to nutlin-3. Moreover, RITA-adapted cells remained generally sensitive to chemotherapy and displayed a substantially lower degree of resistance to irradiation. Again, this is very different to nutlin-3 adaptation that generally and independently of the establishment of a p53 mutation results in a multi-drugresistance phenotype and decreased sensitivity to irradiation. These data demonstrate that drugs that are supposed to share closely related modes of action (i.e., interference with the interaction of $\mathrm{p} 53$ and MDM2) may substantially differ in their effects on cancer cells. Moreover, the use of RITA rather than nutlin-3 for first-line treatment appears favourable because a developing resistance to RITA seems to leave open a larger number of additional treatment options.

\section{Materials and Methods}

Drugs. Nutlin-3 was purchased from Selleck Chemicals via BIOZOL GmbH (Eching, Germany). VCR and CDDP were obtained from TEVA GmbH (Radebeul, Germany). RITA was received from Merck Chemicals (Darmstadt, Germany).

Cell lines. The N-myc-amplified neuroblastoma cell line UKF-NB-3 was established from stage 4 neuroblastoma patients. ${ }^{9}$ The nutlin-3-resistant sublines and the cytotoxic drug-adapted sub-lines (UKF-NB-3 ${ }^{\mathrm{r}} \mathrm{CDDP}^{1000}$, adapted to

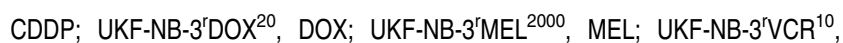
VCR) were derived from the resistant cancer cell line collection and had been established as described. ${ }^{7,9,17}$ UKF-NB-3 cells were adapted to growth in the presence of RITA by continuous exposure to the increasing RITA concentrations by described methods. ${ }^{7,9,17}$

All cells were propagated in Iscove's modified Dulbecco's medium supplemented with $10 \%$ foetal bovine serum, $100 \mathrm{IU} / \mathrm{ml}$ penicillin and $100 \mathrm{mg} / \mathrm{ml}$ streptomycin at $37^{\circ} \mathrm{C}$. Cells were routinely tested for mycoplasma contamination and authenticated by short tandem repeat profiling.

Viability assay. Cell viability was tested by the MTT dye reduction assay after $120 \mathrm{~h}$ incubation modified as described before. ${ }^{7,9,17}$

Irradiation procedure. A total of $10^{4}$ cells were irradiated at room temperature in 96-well cell culture plates (Greiner, Bio-ONE GmbH, Frickenhausen, Germany) with single doses of X-rays ranging from 1 to $10 \mathrm{~Gy}$ using a linear accelerator (SL 75/5, Elekta, Crawley, UK) with $6 \mathrm{MeV}$ photons $/ 100 \mathrm{~cm}$ focus-surface distance with a dose rate of $4.0 \mathrm{~Gy} / \mathrm{min}$. Sham-irradiated cultures were kept at room temperature in the X-ray control room while the other samples were irradiated.

Western blot. Cells were lysed in Triton X-sample buffer and separated by SDS-PAGE. Nuclear extract were prepared using the Nuclear Extract Kit (Active Motif, Carlsbad, CA, USA) following the manufacturer's instruction. Proteins were detected using specific antibodies against $\beta$-actin (BioVision via BioCat $\mathrm{GmbH}$, Heidelberg, Germany), Bcl-2, Bcl-XL, Mcl-1, Bax, XIAP, clAP1, phosphorylated p53 (ser15), cPARP (Asp214), PUMA (all from Cell Signaling via New England Biolabs, frankfurt am Main, Germany), clAP2 (Millipore, Schwalbach, Germany), survivin (R\&D, Wiesbaden, Germany), and p53 (Enzo Life Sciences, Lörrach, Germany) and were visualised by enhanced chemiluminescence using a commercially available kit (Amersham, Freiburg, Germany).

Caspase 3/7 activation. Caspase activation was measured using the Caspase-Glo 3/7 Assay (Promega, Mannheim, Germany) following the manufacturer's instructions.

Mutation analysis of p53. TP53 gene sequencing on CDNAs was performed using the following four pairs of primers: TP53 Ex2-3-f $5^{\prime}$-GTGAC ACGCTTCCCTGGAT-3' and TP53 Ex2-3-r 5'-TCATCTGGACCTGGGTCTTC-3'; TP53 Ex4-5-f 5'-CCCTTCCCAGAAAACCTACC-3' and TP53 Ex4-5-r 5'-CTC CGTCATGTGCTGTGACT-3'; TP53 EX6-7f $5^{\prime}$-GTGCAGCTGTGGGTTGATT-3' and TP53 Ex6-7r 5'-GGTGGTACAGTCAGAGCCAAC-3'; Tp53 Ex8-9-f 5'-CCT CACCATCATCACACTGG-3' and TP53 Ex8-9-r $5^{\prime}$-GTCTGGTCCTGAAGGGT GAA- $3^{\prime}$. In addition all cell lines were examined for TP53 mutations by sequence analysis of genomic DNA as described previously. ${ }^{18}$ PCR was performed as described before. ${ }^{18}$ Each amplicon was sequenced bidirectionally.

Transcriptomics. Triplicates of UKF-NB-3, UKF-NB-3 $3^{r}$ Nutlin $^{10} \mu \mathrm{M}_{\|}$, UKF-NB$3^{r}$ Nutlin $^{10 \mu \mathrm{M} V} \mathrm{~V}, \quad$ UKF-NB-3 $3^{r}$ Nutlin ${ }^{10 \mu \mathrm{M}} \mathrm{VI}, \quad$ UKF-NB-3 ${ }^{\mathrm{r}}$ Nutlin ${ }^{10 \mu \mathrm{M}} \mathrm{VIII}, \quad$ UKF-NB-

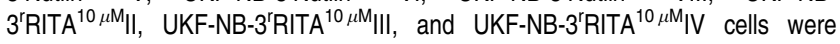
analysed for global cellular gene expression at the mRNA level using Human Gene 1.1 ST microarrays (Affymetrix, Santa Clara, CA, USA) by the Kompetenzzentrum Fluoreszente Bioanalytik, Universität Regensburg (Regensburg, Germany). mRNA was isolated using the RNeasy kit (Qiagen, Hilden, Germany) according to the manufacturer's instructions.

Expression data were processed using the R/bioconductor packages ' $g$ crma' and 'limma' (www.r-project.org/; www.bioconductor.org/) in order to detect differentially expressed genes. ${ }^{19}$ The results were corrected for multiple testing to control the FDR using the approach of Benjamini and Hochberg. ${ }^{20}$ Hierarchical clustering analysis was also performed using $R$.

\section{Conflict of Interest}

The authors declare no conflict of interest.

Acknowledgements. We thank Kristoffer Weber and Boris Fehse (Forschungsabteilung Zell-und Gentherapie, Interdisziplinäre Klinik und Poliklinik für Stammzelltransplantation, Universitätsklinikum Hamburg-Eppendorf) for provision of and support with the lentiviral vectors used. Moreover, we thank Gesa Meincke, 
Eva Bechtold, and Sebastian Grothe for technical support. The work was supported by the charity Hilfe für krebskranke Kinder Frankfurt e.V. and the Frankfurter Stiftung für krebskranke Kinder.

1. Brown CJ, Cheok CF, Verma CS, Lane DP. Reactivation of p53: from peptides to small molecules. Trends Pharmacol Sci 2011; 32: 53-62.

2. Yuan Y, Liao YM, Hsueh CT, Mirshahidi HR. Novel targeted therapeutics: inhibitors of MDM2, ALK and PARP. J Hematol Oncol 2011; 4: 16.

3. Yang J, Ahmed A, Poon E, Perusinghe N, de Haven Brandon A, Box G et al. Smallmolecule activation of $p 53$ blocks hypoxia-inducible factor 1alpha and vascular endothelia growth factor expression in vivo and leads to tumor cell apoptosis in normoxia and hypoxia. Mol Cell Biol 2009; 29: 2243-2253.

4. Verma R, Rigatti MJ, Belinsky GS, Godman CA, Giardina C. DNA damage response to the Mdm2 inhibitor nutlin-3. Biochem Pharmacol 2010; 79: 565-574.

5. Valentine JM, Kumar S, Moumen A. A p53-independent role for the MDM2 antagonist Nutlin-3 in DNA damage response initiation. BMC Cancer 2011; $11: 79$.

6. Aziz MH, Shen H, Maki CG. Acquisition of p53 mutations in response to the non-genotoxic p53 activator Nutlin-3. Oncogene 2011; 30: 4678-4686.

7. Michaelis M, Rothweiler F, Barth S, Cinatl J, van Rikxoort M, Löschmann N et al. Adaptation of cancer cells from different entities to the MDM2 inhibitor nutlin-3 results in the emergence of p53-mutated multi-drug resistant cancer cells. Cell Death Dis 2011; 2: e243.

8. Shen H, Moran DM, Maki CG. Transient nutlin-3a treatment promotes endoreduplication and the generation of therapy-resistant tetraploid cells. Cancer Res 2008; 68: 8260-8268.

9. Kotchetkov R, Driever PH, Cinatl J, Michaelis M, Karaskova J, Blaheta R et al. Increased malignant behavior in neuroblastoma cells with acquired multi-drug resistance does not depend on P-gp expression. Int J Oncol 2005; 27: 1029-1037.

10. Kandel R, Li SQ, Ozcelik H, Rohan T. p53 protein accumulation and mutations in norma and benign breast tissue. Int $J$ Cancer 2000; 87: 73-78.

11. Kato S, Han SY, Liu W, Otsuka K, Shibata H, Kanamaru R et al. Understanding the function-structure and function-mutation relationships of p53 tumor suppressor protein by high-resolution missense mutation analysis. Proc Natl Acad Sci USA 2003; 100 : 8424-8429.
12. Issaeva N, Bozko $P$, Enge M, Protopopova M, Verhoef LG, Masucci $M$ et al. Small molecule RITA binds to p53, blocks p53-HDM-2 interaction and activates p53 function in tumors. Nat Med 2004; 10: 1321-1328.

13. Zhao CY, Grinkevich VV, Nikulenkov F, Bao W, Selivanova G. Rescue of the apoptoticinducing function of mutant p53 by small molecule RITA. Cell Cycle 2010; 9: 1847-1855.

14. Ambrosini G, Sambol EB, Carvajal D, Vassilev LT, Singer S, Schwartz GK. Mouse double minute antagonist Nutlin-3a enhances chemotherapy-induced apoptosis in cancer cells with mutant p53 by activating E2F1. Oncogene 2007; 26: 3473-3481.

15. Ray RM, Bhattacharya S, Johnson LR. Mdm2 inhibition induces apoptosis in p53 deficient human colon cancer cells by activating p73- and E2F1-mediated expression of PUMA and Siva-1. Apoptosis 2011; 16: 35-44.

16. Andreotti V, Ciribilli Y, Monti $\mathrm{P}$, Bisio A, Lion M, Jordan J et al. p53 transactivation and the impact of mutations, cofactors and small molecules using a simplified yeast-based screening system. PLoS One 2011; 6: e20643.

17. Michaelis M, Kleinschmidt MC, Barth S, Rothweiler F, Geiler J, Breitling R et al. Anti-cancer effects of artesunate in a panel of chemoresistant neuroblastoma cell lines. Biochem Pharmacol 2010; 79: 130-136.

18. Hayashi Y, Ueki K, Waha A, Wiestler OD, Louis DN, von Deimling A. Association of EGFR gene amplification and CDKN2 (p16/MTS1) gene deletion in glioblastoma multiforme. Brain Pathol 1997; 7: 871-875.

19. Smyth GK. Linear models and empirical Bayes methods for assessing differential expression in microarray experiments. In Statistical Applications in Genetics and Molecular Biology Vol. 3, 2004. No. 1, Article 3.

20. Benjamini $Y$, Hochberg $Y$. Controlling the false discovery rate: a practical and powerful approach to multiple testing. J $R$ Statist Soc B. 1995; 57: 289-300.

Cell Death and Disease is an open-access journal published by Nature Publishing Group. This work is licensed under the Creative Commons Attribution-NonCommercialShare Alike 3.0 Unported License. To view a copy of this license, visit http://creativecommons.org/licenses/by-nc-sa/3.0/

Supplementary Information accompanies the paper on Cell Death and Disease website (http://www.nature.com/cddis) 